\title{
A Linear Functional Equation of Third Order Associated with the Fibonacci Numbers
}

\author{
Soon-Mo Jung ${ }^{1}$ and Michael Th. Rassias ${ }^{2}$ \\ ${ }^{1}$ Mathematics Section, College of Science and Technology, Hongik University, Sejong 339-701, Republic of Korea \\ ${ }^{2}$ Department of Mathematics, ETH-Zürich, Ramistraße 101, 8092 Zürich, Switzerland
}

Correspondence should be addressed to Soon-Mo Jung; smjung@hongik.ac.kr

Received 7 February 2014; Revised 12 June 2014; Accepted 16 June 2014; Published 30 June 2014

Academic Editor: Jesús G. Falset

Copyright (C) 2014 S.-M. Jung and M. Th. Rassias. This is an open access article distributed under the Creative Commons Attribution License, which permits unrestricted use, distribution, and reproduction in any medium, provided the original work is properly cited.

Given a vector space $X$, we investigate the solutions $f: \mathbb{R} \rightarrow X$ of the linear functional equation of third order $f(x)=p f(x-1)+$ $q f(x-2)+r f(x-3)$, which is strongly associated with a well-known identity for the Fibonacci numbers. Moreover, we prove the Hyers-Ulam stability of that equation.

\section{Introduction}

The problem of stability of functional equations was motivated by a question of Ulam [1] and a solution to it by Hyers [2]. Since then, numerous papers have been published on that subject and we refer to [3-6] for more details, some discussions, and further references; for examples of very recent results, see, for example, [7].

In this paper, as usual, $\mathbb{C}, \mathbb{R}, \mathbb{Z}$, and $\mathbb{N}$ stand for the sets of complex numbers, real numbers, integers, and positive integers, respectively. For a nonempty subset $S$ of a vector space, let $\xi: S \rightarrow S$ be a function. Moreover, $\xi^{0}(x)=x$, $\xi^{n+1}(x)=\xi\left(\xi^{n}(x)\right)$, and (only for bijective $\left.\xi\right) \xi^{-n-1}(x)=$ $\xi^{-1}\left(\xi^{-n}(x)\right)$ for $x \in S$ and $n \in \mathbb{N}_{0}:=\mathbb{N} \cup\{0\}$.

Jung has proved in [3] (see also [8]) some results on solutions and Hyers-Ulam stability of the functional equation

$$
f(x)=p f(\xi(x))-q f\left(\xi^{2}(x)\right),
$$

in the case where $S=\mathbb{R}$ and $\xi(x)=x-1$ for $x \in \mathbb{R}$.

If $S:=\mathbb{N}_{0}$ and $p, q \in \mathbb{Z}$, then solutions $x: \mathbb{N}_{0} \rightarrow \mathbb{Z}$ of the difference equation $f(x)=p f(x-1)-q f(x-2)$ are called the Lucas sequences (see, e.g., [9]). In some special cases they are called with specific names, for example, the Fibonacci numbers $(p=1, q=-1, x(0)=0$, and $x(1)=1)$, the Lucas numbers $(p=1, q=-1, x(0)=2$, and $x(1)=1)$, the Pell numbers $(p=2, q=-1, x(0)=0$, and $x(1)=1)$, the
Pell-Lucas (or companion Lucas) numbers $(p=2, q=-1$, $x(0)=2$, and $x(1)=2)$, and the Jacobsthal numbers $(p=1$, $q=-2, x(0)=0$, and $x(1)=1)$.

For some information and further references concerning the functional equations in a single variable, we refer to [1012]. Let us mention yet that the problem of Hyers-Ulam stability of functional equations is connected to the notions of controlled chaos and shadowing (see [13]).

We remark that if $\xi: S \rightarrow S$ is bijective, then (1) can be written in the following equivalent form:

$$
f\left(\eta^{2}(x)\right)=p f(\eta(x))-q f(x)
$$

where $\eta:=\xi^{-1}$

In view of the last remark, the following Hyers-Ulam stability result concerning (1) can be derived from [14, Theorem 2] (see also [15]).

Theorem 1. Let $p, q \in \mathbb{R}$ be given with $q \neq 0$ and let $S$ be a nonempty subset of a vector space. Assume that $a_{1}, a_{2}$ are the complex roots of the quadratic equation $x^{2}-p x+q=0$ with $\left|a_{i}\right| \neq 1$ for $i \in\{1,2\}$. Moreover, assume that $X$ is either a real vector space if $p^{2}-$ $4 q>0$ or a complex vector space if $p^{2}-4 q<0$. 
Let $\xi: S \rightarrow S$ be bijective. If a function $f: S \rightarrow X$ satisfies the inequality

$$
\left\|f(x)-p f(\xi(x))+q f\left(\xi^{2}(x)\right)\right\| \leq \varepsilon
$$

for all $x \in S$ and for some $\varepsilon \geq 0$, then there exists a unique solution $F: S \rightarrow X$ of (1) with

$$
\|f(x)-F(x)\| \leq \frac{\varepsilon}{\left|\left(\left|a_{1}\right|-1\right)\left(\left|a_{2}\right|-1\right)\right|}
$$

for all $x \in S$.

In [16, Theorem 1.4], the method presented in [3] was modified so as to prove a theorem which is a complement of Theorem 1 . Note that, for bijective $\xi$, the following theorem improves the estimation (4) in some cases (e.g., $a_{1}=3 / 2$, $a_{2}=-3 / 2$, or $\left.a_{1}=1 / 2, a_{2}=-1 / 2\right)$. However, in some other situations (e.g., $a_{1}=3, a_{2}=-3$ ), the estimation (4) is better than (5). The following theorem also complements Theorem 1, because $\xi$ can be quite arbitrary in the case of $(\alpha)$.

Theorem 2. Given $p, q \in \mathbb{R}$ with $q \neq 0$, assume that the distinct complex roots $a_{1}, a_{2}$ of the quadratic equation $x^{2}-p x+$ $q=0$ satisfy one of the following two conditions:

$(\alpha)\left|a_{i}\right|<1$ for $i \in\{1,2\}$;

( $\beta)\left|a_{i}\right| \neq 1$ for $i \in\{1,2\}$ and $\xi: S \rightarrow S$ is bijective.

Moreover, assume that $X$ is either a real vector space if $p^{2}-$ $4 q>0$ or a complex vector space if $p^{2}-4 q<0$. If a function $f: S \rightarrow X$ satisfies inequality (3), then there exists a solution $F: S \rightarrow X$ of (1) such that

$$
\|f(x)-F(x)\| \leq \frac{\varepsilon}{\left|a_{1}-a_{2}\right|}\left(\frac{\left|a_{1}\right|}{|| a_{1}|-1|}+\frac{\left|a_{2}\right|}{|| a_{2}|-1|}\right)
$$

for all $x \in S$. Moreover, if the condition $(\beta)$ is true, then the $F$ is the unique solution of (1) satisfying (5).

In this paper, we investigate the solutions of the functional equation

$$
f(x)=p f(x-1)+q f(x-2)+r f(x-3),
$$

where $p, q, r$ are real constants. Moreover, we also prove the Hyers-Ulam stability of that equation. Equation (6) is a kind of linear functional equations of third order because it is of the form

$f(x)=a_{1}(x) f(\xi(x))+a_{2}(x) f\left(\xi^{2}(x)\right)+a_{3}(x) f\left(\xi^{3}(x)\right)$

for the case of $a_{1}(x)=p, a_{2}(x)=q, a_{3}(x)=r$, and $\xi(x)=$ $x-1$.

\section{General Solution}

In the following theorem, we apply [16, Theorem 1.1] for the investigation of general solutions of the functional equation (6).
Theorem 3. Let p, q, $r$ be real constants such that the cubic equation

$$
x^{3}+p x^{2}-q x+r=0
$$

has the following properties:

(i) $\alpha_{1}$ and $\alpha_{2}$ are two distinct nonzero real roots of the cubic equation (8);

(ii) it holds true that either $\left(\alpha_{i}+p\right)^{2}+4 r / \alpha_{i}>0$ for $i \in\{1,2\}$ or $\left(\alpha_{i}+p\right)^{2}+4 r / \alpha_{i}<0$ for $i \in\{1,2\}$.

Let $X$ be either a real vector space if $\left(\alpha_{i}+p\right)^{2}+4 r / \alpha_{i}>0$ for $i \in\{1,2\}$ or a complex vector space if $\left(\alpha_{i}+p\right)^{2}+4 r / \alpha_{i}<0$ for $i \in\{1,2\}$. Then, a function $f: \mathbb{R} \rightarrow X$ is a solution of the functional equation (6) if and only if there exist functions $h_{1}, h_{2}:[-1,1) \rightarrow X$ such that

$$
\begin{aligned}
f(x)= & \frac{\alpha_{1}}{\alpha_{1}-\alpha_{2}} V_{[x]+1} h_{2}(x-[x]) \\
& +\frac{\alpha_{1} r}{\alpha_{2}\left(\alpha_{1}-\alpha_{2}\right)} V_{[x]} h_{2}(x-[x]-1) \\
& -\frac{\alpha_{2}}{\alpha_{1}-\alpha_{2}} U_{[x]+1} h_{1}(x-[x]) \\
& -\frac{\alpha_{2} r}{\alpha_{1}\left(\alpha_{1}-\alpha_{2}\right)} U_{[x]} h_{1}(x-[x]-1),
\end{aligned}
$$

where $[x]$ denotes the largest integer not exceeding $x$, and $U_{n}$, $V_{n}$ are defined in (13) and (23).

Proof. Assume that $f: \mathbb{R} \rightarrow X$ is a solution of (6). If we define an auxiliary function $g_{1}: \mathbb{R} \rightarrow X$ by

$$
g_{1}(x):=f(x)+\alpha_{1} f(x-1),
$$

then it follows from (6) that $g_{1}$ satisfies

$$
g_{1}(x)=\left(\alpha_{1}+p\right) g_{1}(x-1)+\frac{r}{\alpha_{1}} g_{1}(x-2)
$$

for any $x \in \mathbb{R}$. According to [16, Theorem 1.1] or [3, Theorem 2.1], there exists a function $h_{1}:[-1,1) \rightarrow X$ such that

$$
\begin{aligned}
g_{1}(x) & =f(x)+\alpha_{1} f(x-1) \\
& =U_{[x]+1} h_{1}(x-[x])+\frac{r}{\alpha_{1}} U_{[x]} h_{1}(x-[x]-1)
\end{aligned}
$$

for all $x \in \mathbb{R}$, where

$$
U_{n}=\frac{a^{n}-b^{n}}{a-b} \quad(n \in \mathbb{Z})
$$

and $a, b$ are the distinct roots of the quadratic equation

$$
x^{2}-\left(\alpha_{1}+p\right) x-\frac{r}{\alpha_{1}}=0
$$

that is,

$$
\begin{aligned}
& a=\frac{\alpha_{1}+p}{2}+\sqrt{\left(\frac{\alpha_{1}+p}{2}\right)^{2}+\frac{r}{\alpha_{1}}}, \\
& b=\frac{\alpha_{1}+p}{2}-\sqrt{\left(\frac{\alpha_{1}+p}{2}\right)^{2}+\frac{r}{\alpha_{1}}} .
\end{aligned}
$$


Since $a$ is a root of the quadratic equation (14), we have

$$
a^{2}=\left(\alpha_{1}+p\right) a+\frac{r}{\alpha_{1}} \text {. }
$$

We multiply both sides of (16) with $a$ and make use of (16) and (i) to get

$$
\begin{aligned}
a^{3} & =p a^{2}+\alpha_{1} a^{2}+\frac{r}{\alpha_{1}} a \\
& =p a^{2}+\alpha_{1}\left(\left(\alpha_{1}+p\right) a+\frac{r}{\alpha_{1}}\right)+\frac{r}{\alpha_{1}} a \\
& =p a^{2}+\frac{a}{\alpha_{1}}\left(\alpha_{1}^{3}+p \alpha_{1}^{2}+r\right)+r \\
& =p a^{2}+q a+r .
\end{aligned}
$$

Similarly, we also obtain

$$
b^{3}=p b^{2}+q b+r
$$

Using (13), (17), and (18), we have

$$
\begin{aligned}
& p U_{n-1}+q U_{n-2}+r U_{n-3} \\
&= \frac{\left(p a^{2}+q a+r\right) a^{n-3}-\left(p b^{2}+q b+r\right) b^{n-3}}{a-b} \\
&= \frac{a^{n}-b^{n}}{a-b}=U_{n}
\end{aligned}
$$

for all $n \in \mathbb{Z}$.

If we define an auxiliary function $g_{2}: \mathbb{R} \rightarrow X$ by

$$
g_{2}(x):=f(x)+\alpha_{2} f(x-1),
$$

then it follows from (6) that $g_{2}$ satisfies

$$
g_{2}(x)=\left(\alpha_{2}+p\right) g_{2}(x-1)+\frac{r}{\alpha_{2}} g_{2}(x-2)
$$

for any $x \in \mathbb{R}$. According to [16, Theorem 1.1] or [3, Theorem 2.1], there exists a function $h_{2}:[-1,1) \rightarrow X$ such that

$$
\begin{aligned}
g_{2}(x) & =f(x)+\alpha_{2} f(x-1) \\
& =V_{[x]+1} h_{2}(x-[x])+\frac{r}{\alpha_{2}} V_{[x]} h_{2}(x-[x]-1)
\end{aligned}
$$

for all $x \in \mathbb{R}$, where

$$
V_{n}=\frac{c^{n}-d^{n}}{c-d} \quad(n \in \mathbb{Z})
$$

and $c, d$ are the distinct roots of the quadratic equation

$$
x^{2}-\left(\alpha_{2}+p\right) x-\frac{r}{\alpha_{2}}=0
$$

that is,

$$
\begin{aligned}
& c=\frac{\alpha_{2}+p}{2}+\sqrt{\left(\frac{\alpha_{2}+p}{2}\right)^{2}+\frac{r}{\alpha_{2}}}, \\
& d=\frac{\alpha_{2}+p}{2}-\sqrt{\left(\frac{\alpha_{2}+p}{2}\right)^{2}+\frac{r}{\alpha_{2}}} .
\end{aligned}
$$

As in the first part, we verify that

$$
V_{n}=p V_{n-1}+q V_{n-2}+r V_{n-3}
$$

for all $n \in \mathbb{Z}$.

We now multiply (12) with $\alpha_{2}$ and (22) with $\alpha_{1}$, we subtract the former from the latter, and we then divide the resulting equation by $\left(\alpha_{1}-\alpha_{2}\right)$ to get (9).

We assume that a function $f: \mathbb{R} \rightarrow X$ is given by (9), where $h_{1}, h_{2}:[-1,1) \rightarrow X$ are arbitrarily given functions and $U_{n}, V_{n}$ are given by (13) and (23), respectively. Then, by (9), (19), and (26), we have

$$
\begin{aligned}
p f(x & -1)+q f(x-2)+r f(x-3) \\
= & \frac{\alpha_{1}}{\alpha_{1}-\alpha_{2}}\left(p V_{[x]}+q V_{[x]-1}+r V_{[x]-2}\right) h_{2}(x-[x]) \\
& +\frac{\alpha_{1} r}{\alpha_{2}\left(\alpha_{1}-\alpha_{2}\right)}\left(p V_{[x]-1}+q V_{[x]-2}+r V_{[x]-3}\right) \\
& \times h_{2}(x-[x]-1) \\
& -\frac{\alpha_{2}}{\alpha_{1}-\alpha_{2}}\left(p U_{[x]}+q U_{[x]-1}+r U_{[x]-2}\right) h_{1}(x-[x]) \\
& -\frac{\alpha_{2} r}{\alpha_{1}\left(\alpha_{1}-\alpha_{2}\right)}\left(p U_{[x]-1}+q U_{[x]-2}+r U_{[x]-3}\right) \\
& \times h_{1}(x-[x]-1) \\
= & \frac{\alpha_{1}}{\alpha_{1}-\alpha_{2}} V_{[x]+1} h_{2}(x-[x]) \\
& +\frac{\alpha_{1} r}{\alpha_{2}\left(\alpha_{1}-\alpha_{2}\right)} V_{[x]} h_{2}(x-[x]-1) \\
& -\frac{\alpha_{2}}{\alpha_{1}-\alpha_{2}} U_{[x]+1} h_{1}(x-[x]) \\
& -\frac{\alpha_{2} r}{\alpha_{1}\left(\alpha_{1}-\alpha_{2}\right)} U_{[x]} h_{1}(x-[x]-1)=f(x)
\end{aligned}
$$

for all $x \in \mathbb{R}$, which implies that $f$ is a solution of (6).

According to $\left[17\right.$, p. 92], the Fibonacci numbers $F_{n}$ satisfy the identity

$$
F_{n}^{2}=2 F_{n-1}^{2}+2 F_{n-2}^{2}-F_{n-3}^{2}
$$

for all integers $n>3$. We can easily notice that the linear equation of third order

$$
f(x)=2 f(x-1)+2 f(x-2)-f(x-3)
$$

is strongly related to identity (28). 
Corollary 4. Let $X$ be a real vector space. A function $f: \mathbb{R} \rightarrow$ $X$ is a solution of the functional equation (29) if and only if there exist functions $h_{1}, h_{2}:[-1,1) \rightarrow X$ such that

$$
\begin{aligned}
f(x)= & \frac{5+3 \sqrt{5}}{10} U_{[x]+1} h_{1}(x-[x]) \\
& +\frac{15+7 \sqrt{5}}{10} U_{[x]} h_{1}(x-[x]-1) \\
& +\frac{5-3 \sqrt{5}}{10} V_{[x]+1} h_{2}(x-[x]) \\
& +\frac{15-7 \sqrt{5}}{10} V_{[x]} h_{2}(x-[x]-1),
\end{aligned}
$$

where $U_{n}$ and $V_{n}$ are defined in (33).

Proof. If we set $p=2, q=2$, and $r=-1$ in (8), then the cubic equation

$$
x^{3}+2 x^{2}-2 x-1=0
$$

has three distinct nonzero roots including

$$
\alpha_{1}=-\frac{3}{2}+\frac{\sqrt{5}}{2}, \quad \alpha_{2}=-\frac{3}{2}-\frac{\sqrt{5}}{2} .
$$

Moreover, it holds that $\left(\alpha_{1}+p\right)^{2}+4 r / \alpha_{1}>0$ and $\left(\alpha_{2}+p\right)^{2}+$ $4 r / \alpha_{2}>0$. By (13), (15), (23), and (25), we have

$$
U_{n}=\frac{a^{n}-b^{n}}{a-b}, \quad V_{n}=\frac{c^{n}-d^{n}}{c-d},
$$

where we make use of (15) and (25) to calculate

$$
a=\frac{3+\sqrt{5}}{2}, \quad b=-1, \quad c=\frac{3-\sqrt{5}}{2}, \quad d=-1 .
$$

Finally, in view of Theorem 3, we conclude that the assertion of our corollary is true.

Corollary 5. If a function $f: \mathbb{R} \rightarrow \mathbb{R}$ is a solution of functional equation (29), then there exist real constants $\mu_{1}, \mu_{2}$, $v_{1}$, and $v_{2}$ such that

$$
\begin{aligned}
f(n)= & \frac{5+3 \sqrt{5}}{10} \mu_{1} U_{n+1}+\frac{15+7 \sqrt{5}}{10} \mu_{2} U_{n} \\
& +\frac{5-3 \sqrt{5}}{10} \nu_{1} V_{n+1}+\frac{15-7 \sqrt{5}}{10} \nu_{2} V_{n}
\end{aligned}
$$

for all $n \in \mathbb{Z}$, where $U_{n}$ and $V_{n}$ are defined in (33).

\section{Hyers-Ulam Stability}

We apply the classical direct method to the proof of the following theorem. The classical direct method was first proposed by Hyers [2].

Theorem 6. Let $p, q, r$ be real constants with $r \neq 0$, let $\alpha$ be a nonzero root of the cubic equation (8), and let $a, b$ be the roots of the quadratic equation $x^{2}-(\alpha+p) x-r / \alpha=0$ with $|a|>1$ and $0<|b|<1$. Let $X$ be either a real Banach space if $(\alpha+p)^{2}+$ $4 r / \alpha>0$ or a complex Banach space if $(\alpha+p)^{2}+4 r / \alpha<0$. If a function $f: \mathbb{R} \rightarrow X$ satisfies the inequality

$$
\|f(x)-p f(x-1)-q f(x-2)-r f(x-3)\| \leq \varepsilon
$$

for all $x \in \mathbb{R}$ and for some $\varepsilon \geq 0$, then there exists a solution $G: \mathbb{R} \rightarrow X$ of (6) such that

$$
\|f(x)+\alpha f(x-1)-G(x)\| \leq \frac{|a|-|b|}{|a-b|} \frac{\varepsilon}{(|a|-1)(1-|b|)}
$$

for all $x \in \mathbb{R}$.

Proof. If we define an auxiliary function $g: \mathbb{R} \rightarrow X$ by

$$
g(x):=f(x)+\alpha f(x-1),
$$

then, as we did in (11), it follows from (36) that $g$ satisfies the inequality

$$
\left\|g(x)-(\alpha+p) g(x-1)-\frac{r}{\alpha} g(x-2)\right\| \leq \varepsilon
$$

or

$$
\|g(x)-a g(x-1)-b[g(x-1)-a g(x-2)]\| \leq \varepsilon
$$

for any $x \in \mathbb{R}$. have

If we replace $x$ with $x-k$ in the last inequality, then we

$$
\begin{aligned}
& \| g(x-k)-a g(x-k-1) \\
& -b[g(x-k-1)-a g(x-k-2)] \| \leq \varepsilon
\end{aligned}
$$

for all $x \in \mathbb{R}$. Furthermore, we get

$$
\begin{aligned}
& \| b^{k}[g(x-k)-a g(x-k-1)] \\
& -b^{k+1}[g(x-k-1)-a g(x-k-2)] \| \leq|b|^{k} \varepsilon
\end{aligned}
$$

for all $x \in \mathbb{R}$ and $k \in \mathbb{Z}$. By (42), we obviously have

$$
\begin{aligned}
& \left\|g(x)-a g(x-1)-b^{n}[g(x-n)-a g(x-n-1)]\right\| \\
& \leq \sum_{k=0}^{n-1} \| b^{k}[g(x-k)-a g(x-k-1)] \\
& \quad-b^{k+1}[g(x-k-1)-a g(x-k-2)] \| \\
& \leq \sum_{k=0}^{n-1}|b|^{k} \varepsilon
\end{aligned}
$$

for $x \in \mathbb{R}$ and $n \in \mathbb{N}$.

For any $x \in \mathbb{R},(42)$ implies that the sequence $\left\{b^{n}[g(x-\right.$ $n)-a g(x-n-1)]\}$ is a Cauchy sequence (note that $0<|b|<1$ ). Therefore, we can define a function $G_{1}: \mathbb{R} \rightarrow X$ by

$$
G_{1}(x):=\lim _{n \rightarrow \infty} b^{n}[g(x-n)-a g(x-n-1)],
$$


since $X$ is complete. In view of the definition of $G_{1}$ and using the relations, $a+b=\alpha+p$ and $a b=-r / \alpha$, we obtain

$$
\begin{aligned}
(\alpha+p) G_{1}(x-1)+\frac{r}{\alpha} G_{1}(x-2) \\
=(a+b) G_{1}(x-1)-a b G_{1}(x-2) \\
=\frac{a+b}{b} \lim _{n \rightarrow \infty} b^{n+1}[g(x-(n+1))-a g(x-(n+1)-1)] \\
\quad-\frac{a b}{b^{2}} \lim _{n \rightarrow \infty} b^{n+2}[g(x-(n+2))-a g(x-(n+2)-1)] \\
=\frac{a+b}{b} G_{1}(x)-\frac{a}{b} G_{1}(x)=G_{1}(x)
\end{aligned}
$$

for all $x \in \mathbb{R}$. Since $\alpha$ is a nonzero root of the cubic equation (8), it follows from (45) that

$$
\begin{aligned}
G_{1}(x)-p G_{1}(x-1)-q G_{1}(x-2)-r G_{1}(x-3) \\
=(\alpha+p) G_{1}(x-1)+\frac{r}{\alpha} G_{1}(x-2)-p G_{1}(x-1) \\
\quad-q G_{1}(x-2)-r G_{1}(x-3) \\
=\alpha G_{1}(x-1)+\left(-q+\frac{r}{\alpha}\right) G_{1}(x-2)-r G_{1}(x-3) \\
=\alpha G_{1}(x-1)+\left(-\alpha^{2}-p \alpha\right) G_{1}(x-2)-r G_{1}(x-3) \\
=\alpha\left((\alpha+p) G_{1}(x-2)+\frac{r}{\alpha} G_{1}(x-3)\right) \\
\quad-\alpha(\alpha+p) G_{1}(x-2)-r G_{1}(x-3)=0
\end{aligned}
$$

for all $x \in \mathbb{R}$. Hence, we conclude that $G_{1}$ is a solution of (6).

If $n$ tends to infinity, then (43) yields that

$$
\left\|g(x)-a g(x-1)-G_{1}(x)\right\| \leq \frac{\varepsilon}{1-|b|}
$$

for every $x \in \mathbb{R}$.

On the other hand, it also follows from (36) that

$$
\|g(x)-b g(x-1)-a[g(x-1)-b g(x-2)]\| \leq \varepsilon
$$

for all $x \in \mathbb{R}$. Analogously to (42), replacing $x$ by $x+k$ in the last inequality and then dividing by $|a|^{k}$ both sides of the resulting inequality, then we have

$$
\begin{aligned}
& \| a^{-k}[g(x+k)-b g(x+k-1)] \\
& \quad-a^{-k+1}[g(x+k-1)-b g(x+k-2)] \| \leq|a|^{-k} \varepsilon
\end{aligned}
$$

for all $x \in \mathbb{R}$ and $k \in \mathbb{Z}$. By using (49), we further obtain

$$
\begin{aligned}
& \left\|a^{-n}[g(x+n)-b g(x+n-1)]-[g(x)-b g(x-1)]\right\| \\
& \leq \sum_{k=1}^{n} \| a^{-k}[g(x+k)-b g(x+k-1)] \\
& \quad-a^{-k+1}[g(x+k-1)-b g(x+k-2)] \| \\
& \leq \sum_{k=1}^{n}|a|^{-k} \mathcal{E}
\end{aligned}
$$

for $x \in \mathbb{R}$ and $n \in \mathbb{N}$.

On account of (49), we see that the sequence $\left\{a^{-n}[g(x+\right.$ $n)-b g(x+n-1)]\}$ is a Cauchy sequence for any fixed $x \in \mathbb{R}$ (note that $|a|>1$ ). Hence, we can define a function $G_{2}: \mathbb{R} \rightarrow$ $X$ by

$$
G_{2}(x):=\lim _{n \rightarrow \infty} a^{-n}[g(x+n)-b g(x+n-1)] .
$$

Due to the definition of $G_{2}$ and the relations, $a+b=\alpha+p$ and $a b=-r / \alpha$, we get

$$
\begin{aligned}
(\alpha+p) G_{2}(x-1)+\frac{r}{\alpha} G_{2}(x-2) \\
=(a+b) G_{2}(x-1)-a b G_{2}(x-2) \\
=\frac{a+b}{a} \lim _{n \rightarrow \infty} a^{-(n-1)}[g(x+n-1)-b g(x+n-2)] \\
\quad-\frac{a b}{a^{2}} \lim _{n \rightarrow \infty} a^{-(n-2)}[g(x+n-2)-b g(x+n-3)] \\
=\frac{a+b}{a} G_{2}(x)-\frac{b}{a} G_{2}(x)=G_{2}(x)
\end{aligned}
$$

for any $x \in \mathbb{R}$. Similarly as in the first part, we can show that $G_{2}$ is a solution of (6).

If we let $n$ tend to infinity, then it follows from (50) that

$$
\left\|G_{2}(x)-g(x)+b g(x-1)\right\| \leq \frac{\varepsilon}{|a|-1}
$$

for $x \in \mathbb{R}$.

It follows from (47) and (53) that

$$
\begin{aligned}
\left\|g(x-1)-\frac{1}{a-b} G_{2}(x)+\frac{1}{a-b} G_{1}(x)\right\| \\
\leq\left\|\frac{1}{a-b} G_{1}(x)-\frac{1}{a-b} g(x)+\frac{a}{a-b} g(x-1)\right\| \\
\quad+\left\|\frac{1}{a-b} g(x)-\frac{b}{a-b} g(x-1)-\frac{1}{a-b} G_{2}(x)\right\| \\
\leq \frac{|a|-|b|}{|a-b|} \frac{\varepsilon}{(|a|-1)(1-|b|)}
\end{aligned}
$$

for any $x \in \mathbb{R}$.

Finally, if we define a function $G: \mathbb{R} \rightarrow X$ by

$$
G(x):=\frac{1}{a-b} G_{2}(x+1)-\frac{1}{a-b} G_{1}(x+1)
$$


for all $x \in \mathbb{R}$, then $G$ is also a solution of (6). Moreover, the validity of (37) follows from the last inequality.

The following theorem is the main theorem of this paper.

Theorem 7. Given real constants $p, q, r$ with $r \neq 0$, let $\alpha_{1}$ and $\alpha_{2}$ be distinct nonzero roots of cubic equation (8) and let $a_{i}, b_{i}$ be the roots of the quadratic equation $x^{2}-\left(\alpha_{i}+p\right) x-r / \alpha_{i}=0$ with $\left|a_{i}\right|>1$ and $0<\left|b_{i}\right|<1$ for $i \in\{1,2\}$. Assume that either $\left(\alpha_{i}+p\right)^{2}+4 r / \alpha_{i}>0$ for all $i \in\{1,2\}$ or $\left(\alpha_{i}+p\right)^{2}+4 r / \alpha_{i}<0$ for all $i \in\{1,2\}$. Let $X$ be either a real Banach space if $\left(\alpha_{i}+p\right)^{2}+$ $4 r / \alpha_{i}>0$ or a complex Banach space if $\left(\alpha_{i}+p\right)^{2}+4 r / \alpha_{i}<0$. If a function $f: \mathbb{R} \rightarrow X$ satisfies inequality (36) for all $x \in \mathbb{R}$ and for some $\varepsilon \geq 0$, then there exists a solution $F: \mathbb{R} \rightarrow X$ of (6) such that

$$
\begin{aligned}
\|f(x)-F(x)\| \leq & \frac{\left|a_{1}\right|-\left|b_{1}\right|}{\left|a_{1}-b_{1}\right|} \frac{\left|\alpha_{2}\right|}{\left|\alpha_{1}-\alpha_{2}\right|} \frac{\varepsilon}{\left(\left|a_{1}\right|-1\right)\left(1-\left|b_{1}\right|\right)} \\
& +\frac{\left|a_{2}\right|-\left|b_{2}\right|}{\left|a_{2}-b_{2}\right|} \frac{\left|\alpha_{1}\right|}{\left|\alpha_{1}-\alpha_{2}\right|} \frac{\varepsilon}{\left(\left|a_{2}\right|-1\right)\left(1-\left|b_{2}\right|\right)}
\end{aligned}
$$

for all $x \in \mathbb{R}$.

Proof. According to Theorem 6, there exists a solution $F_{i}$ : $\mathbb{R} \rightarrow X$ of $(6)$ such that

$$
\left\|f(x)+\alpha_{i} f(x-1)-F_{i}(x)\right\| \leq \frac{\left|a_{i}\right|-\left|b_{i}\right|}{\left|a_{i}-b_{i}\right|} \frac{\varepsilon}{\left(\left|a_{i}\right|-1\right)\left(1-\left|b_{i}\right|\right)}
$$

for any $x \in \mathbb{R}$ and $i \in\{1,2\}$. In view of the last inequalities, we have

$$
\begin{aligned}
&\left\|f(x)-\frac{\alpha_{1}}{\alpha_{1}-\alpha_{2}} F_{2}(x)+\frac{\alpha_{2}}{\alpha_{1}-\alpha_{2}} F_{1}(x)\right\| \\
& \leq\left\|\frac{\alpha_{2}}{\alpha_{1}-\alpha_{2}} F_{1}(x)-\frac{\alpha_{2}}{\alpha_{1}-\alpha_{2}} f(x)-\frac{\alpha_{1} \alpha_{2}}{\alpha_{1}-\alpha_{2}} f(x-1)\right\| \\
& \quad+\left\|\frac{\alpha_{1}}{\alpha_{1}-\alpha_{2}} f(x)+\frac{\alpha_{1} \alpha_{2}}{\alpha_{1}-\alpha_{2}} f(x-1)-\frac{\alpha_{1}}{\alpha_{1}-\alpha_{2}} F_{2}(x)\right\| \\
& \leq \frac{\left|a_{1}\right|-\left|b_{1}\right|}{\left|a_{1}-b_{1}\right|} \frac{\left|\alpha_{2}\right|}{\left|\alpha_{1}-\alpha_{2}\right|} \frac{\varepsilon}{\left(\left|a_{1}\right|-1\right)\left(1-\left|b_{1}\right|\right)} \\
&+\frac{\left|a_{2}\right|-\left|b_{2}\right|}{\left|a_{2}-b_{2}\right|} \frac{\left|\alpha_{1}\right|}{\left|\alpha_{1}-\alpha_{2}\right|} \frac{\varepsilon}{\left(\left|a_{2}\right|-1\right)\left(1-\left|b_{2}\right|\right)}
\end{aligned}
$$

for all $x \in \mathbb{R}$.

If we define a function $F: \mathbb{R} \rightarrow X$ by

$$
F(x):=\frac{\alpha_{1}}{\alpha_{1}-\alpha_{2}} F_{2}(x)-\frac{\alpha_{2}}{\alpha_{1}-\alpha_{2}} F_{1}(x)
$$

for each $x \in \mathbb{R}$, then $F$ is also a solution of (6), and inequality (56) follows from the last inequality.

\section{Conflict of Interests}

The authors declare that there is no conflict of interests regarding the publication of this paper.

\section{Acknowledgments}

This research paper was completed while Soon-Mo Jung was a visiting scholar at National Technical University of Athens during February 2014. He would like to express his cordial thanks to Professor Themistocles M. Rassias for his hospitality and kindness. This research was supported by Basic Science Research Program through the National Research Foundation of Korea (NRF) funded by the Ministry of Education (no. 2013R1A1A2005557). The authors would like to express their cordial thanks to the referees for useful remarks.

\section{References}

[1] S. M. Ulam, A Collection of Mathematical Problems, Interscience, New York, NY, USA, 1960.

[2] D. H. Hyers, "On the stability of the linear functional equation," Proceedings of the National Academy of Sciences of the United States of America, vol. 27, pp. 222-224, 1941.

[3] S.-M. Jung, "Functional equation $f(x)=p f(x-1)-q f(x-$ 2) and its Hyers-Ulam stability," Journal of Inequalities and Applications, vol. 2008, Article ID 181678, 2009.

[4] S.-M. Jung, Hyers-Ulam-Rassias Stability of Functional Equations in Nonlinear Analysis, vol. 48 of Springer Optimization and Its Applications, Springer, New York, NY, USA, 2011.

[5] S.-M. Jung, D. Popa, and M. T. Rassias, "On the stability of the linear functional equation in a single variable on complete metric groups," Journal of Global Optimization, vol. 59, no. 1, pp. 165-171, 2014.

[6] Z. Moszner, "On the stability of functional equations," Aequationes Mathematicae, vol. 77, no. 1-2, pp. 33-88, 2009.

[7] N. Brillouet-Belluot, J. Brzdek, and K. Cieplinski, "On some recent developments in Ulam's type stability," Abstract and Applied Analysis, vol. 2012, Article ID 716936, 41 pages, 2012.

[8] S.-M. Jung, "Hyers-Ulam stability of Fibonacci functional equation," Bulletin of the Iranian Mathematical Society, vol. 35, no. 2, pp. 217-227, 2009.

[9] P. Ribenboim, My Numbers, My Friends: Popular Lectures on Number Theory, Springer, New York, NY, USA, 2000.

[10] K. Baron and W. Jarczyk, "Recent results on functional equations in a single variable, perspectives and open problems," Aequationes Mathematicae, vol. 61, no. 1-2, pp. 1-48, 2001.

[11] M. Kuczma, Functional Equations in a Single Variable, Polish Scientific, Warszawa, Poland, 1968.

[12] M. Kuczma, B. Choczewski, and R. Ger, Iterative Functional Equations, vol. 32 of Encyclopedia of Mathematics and Its Applications, Cambridge University Press, Cambridge, UK, 1990.

[13] S. Y. Pilyugin, Shadowing in Dynamical Systems, vol. 1706 of Lecture Notes in Mathematics, Springer, Berlin, Germany, 1999.

[14] J. Brzdek, D. Popa, and B. Xu, "Hyers-Ulam stability for linear equations of higher orders," Acta Mathematica Hungarica, vol. 120, no. 1-2, pp. 1-8, 2008.

[15] T. Trif, "Hyers-Ulam-Rassias stability of a linear functional equation with constant coefficients," Nonlinear Functional Analysis and Applications, vol. 11, no. 5, pp. 881-889, 2006. 
[16] J. Brzdek and S.-M. Jung, "A note on stability of a linear functional equation of second order connected with the Fibonacci numbers and Lucas sequences," Journal of Inequalities and Applications, vol. 2010, Article ID 793947, 10 pages, 2010.

[17] T. Koshy, Fibonacci and Lucas Numbers with Applications, John Wiley \& Sons, New York, NY, USA, 2001. 


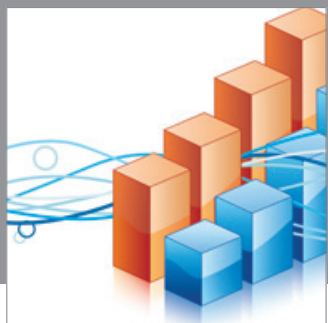

Advances in

Operations Research

mansans

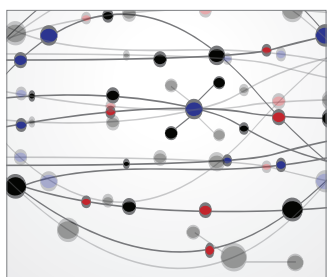

The Scientific World Journal
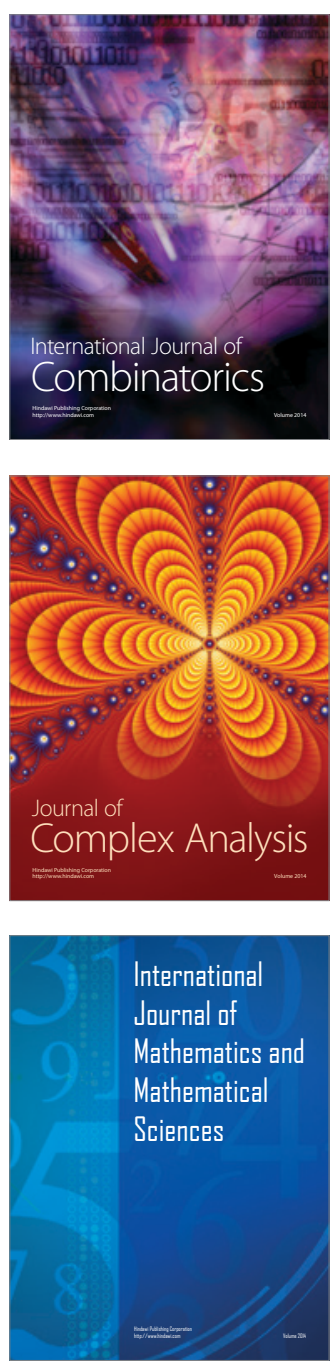
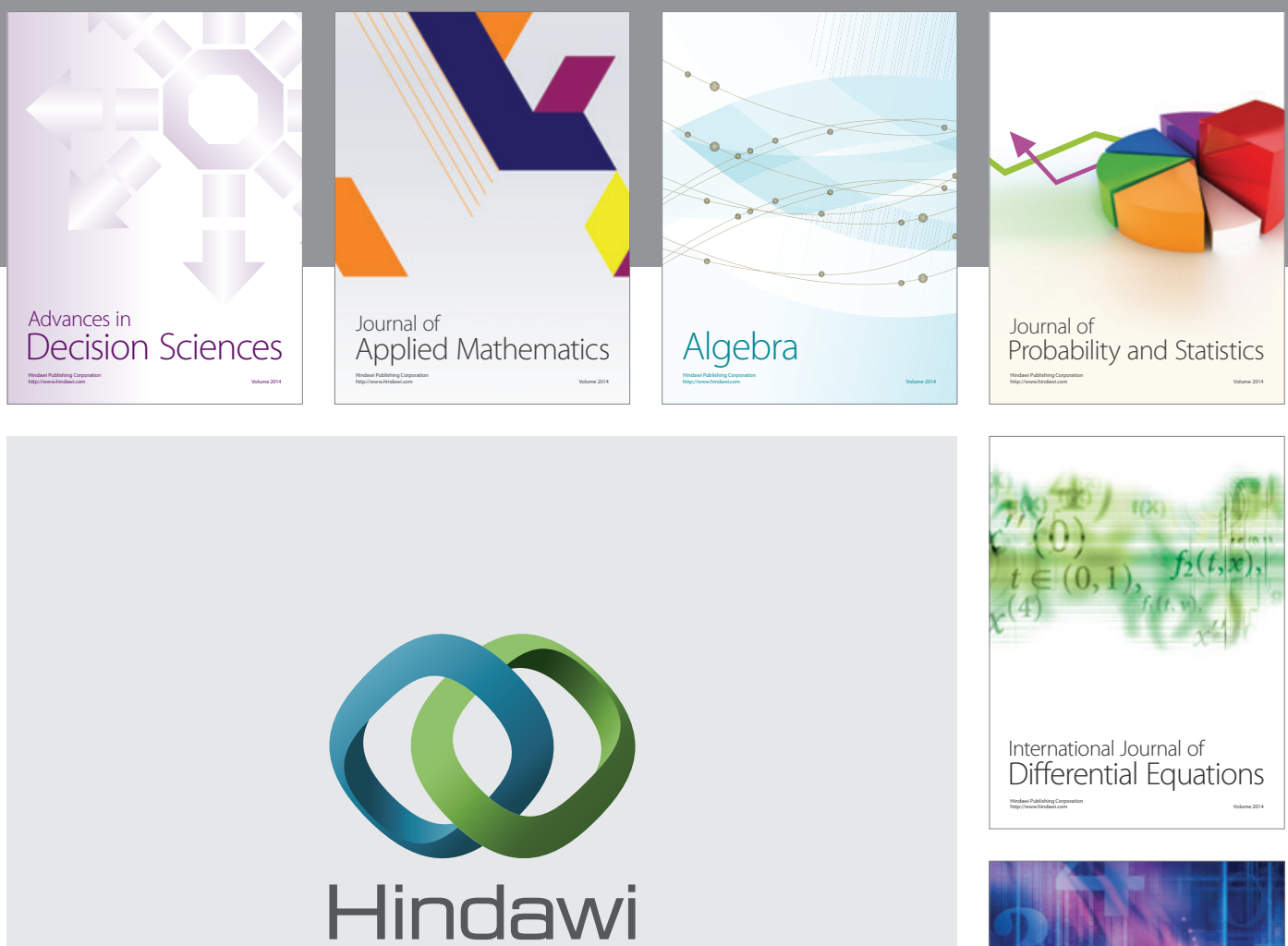

Submit your manuscripts at http://www.hindawi.com
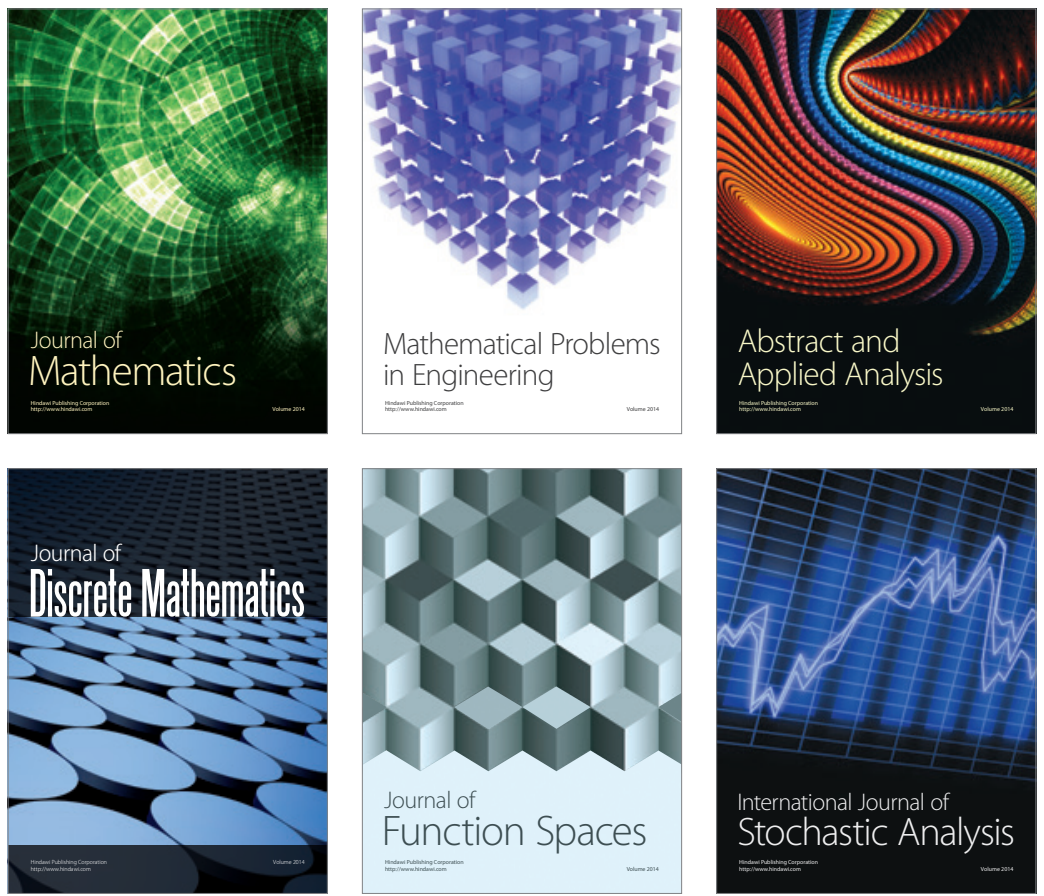

Journal of

Function Spaces

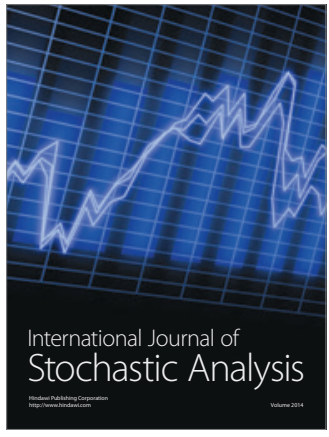

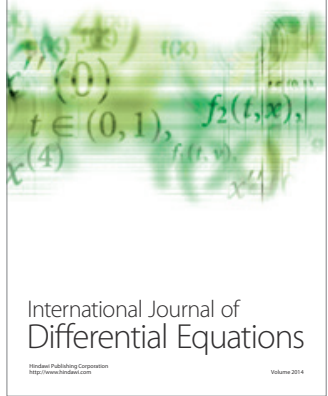
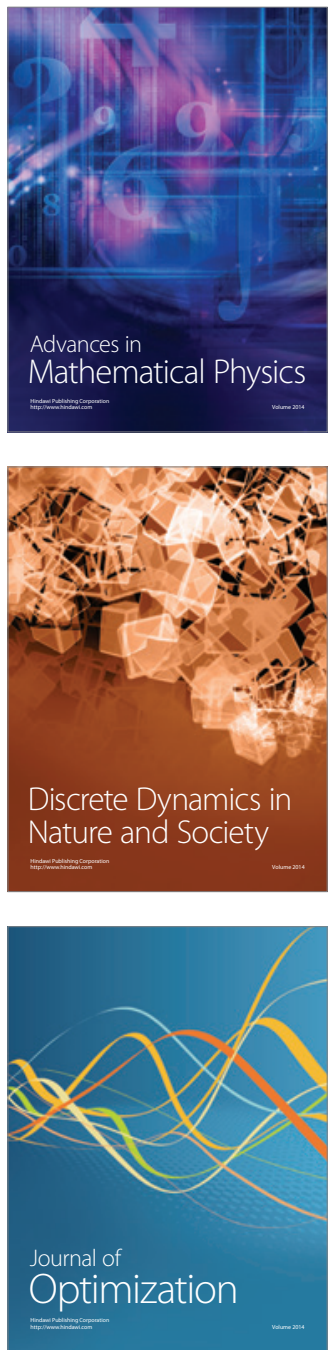